\title{
Hybrid Copper-Silver Conductive Tracks for Enhanced Oxidation Resistance under Flash Light Sintering
}

\author{
Changyong Yim", Allen Sandwell, and Simon S. Park ${ }^{*}$
}

Department of Mechanical and Manufacturing Engineering, University of Calgary, Calgary, Alberta T2N 1N4, Canada

* Author to whom correspondence should be addressed.

E-mail: changyong.vim@ucalgary.ca, simon.park@ucalgary.ca 


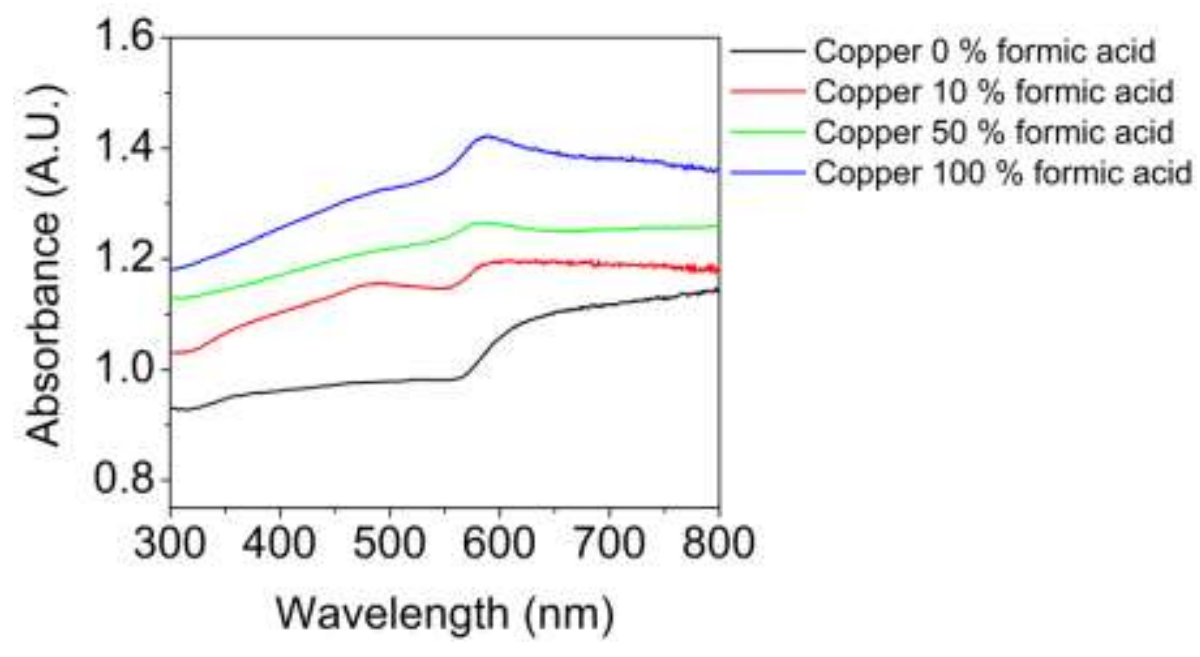

Figure S1. CuNPs were treated with various concentration of formic acid aqueous solution: $0 \%$ (black), 10\% (red), 50\% (green), and 100\% (blue). As the concentration of formic acid increased, the absorbance peak of the UV-vis spectrum at $580 \mathrm{~nm}$ (corresponding to CuNPs) became more apparent. This implies that the copper oxide was successfully removed by formic acid. ${ }^{1}$ 

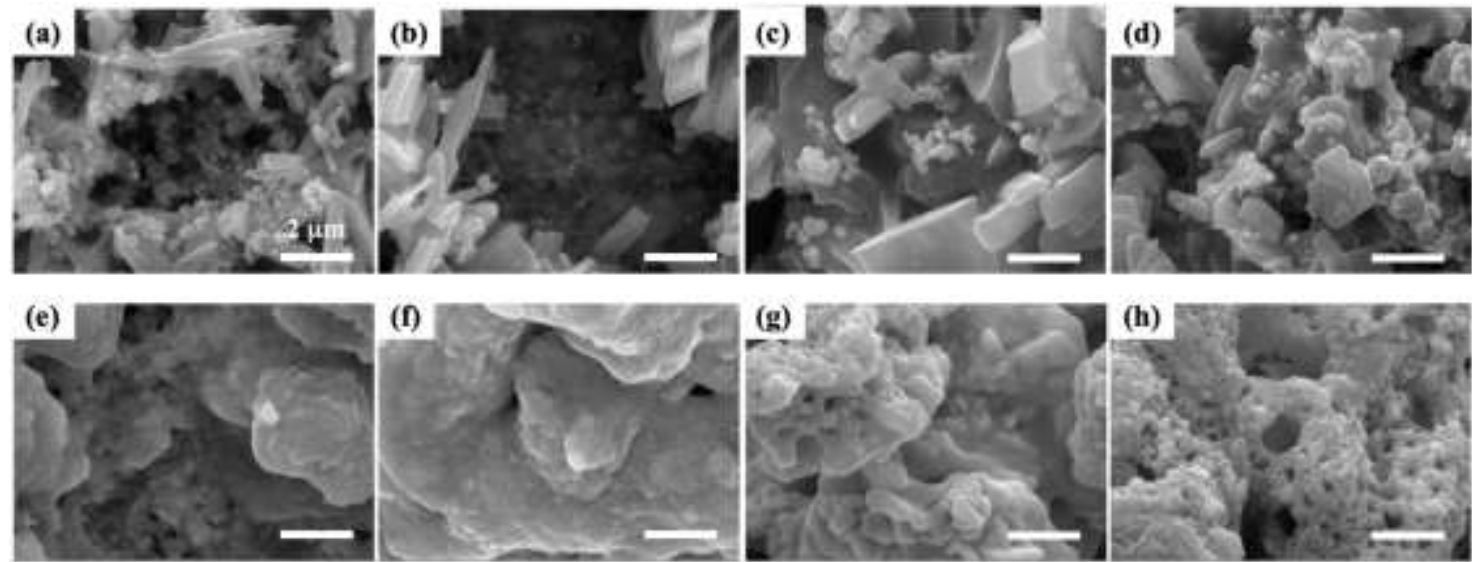

(g)

(h)

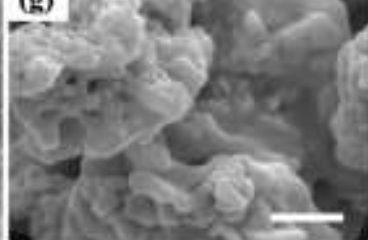

A.

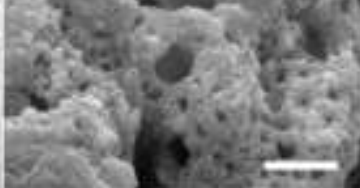

Figure S2. SEM images of the F-CuAg films with various concentrations of $\mathrm{AgNO}_{3}$. (a),(e) F-CuAg-00 (b),(f) F-CuAg-01 (c),(g) F-CuAg-02, and (d),(h) F-CuAg-03. (a-d): before flash, (e-h): after flash. The scale bar represents $2 \mu \mathrm{m}$. 

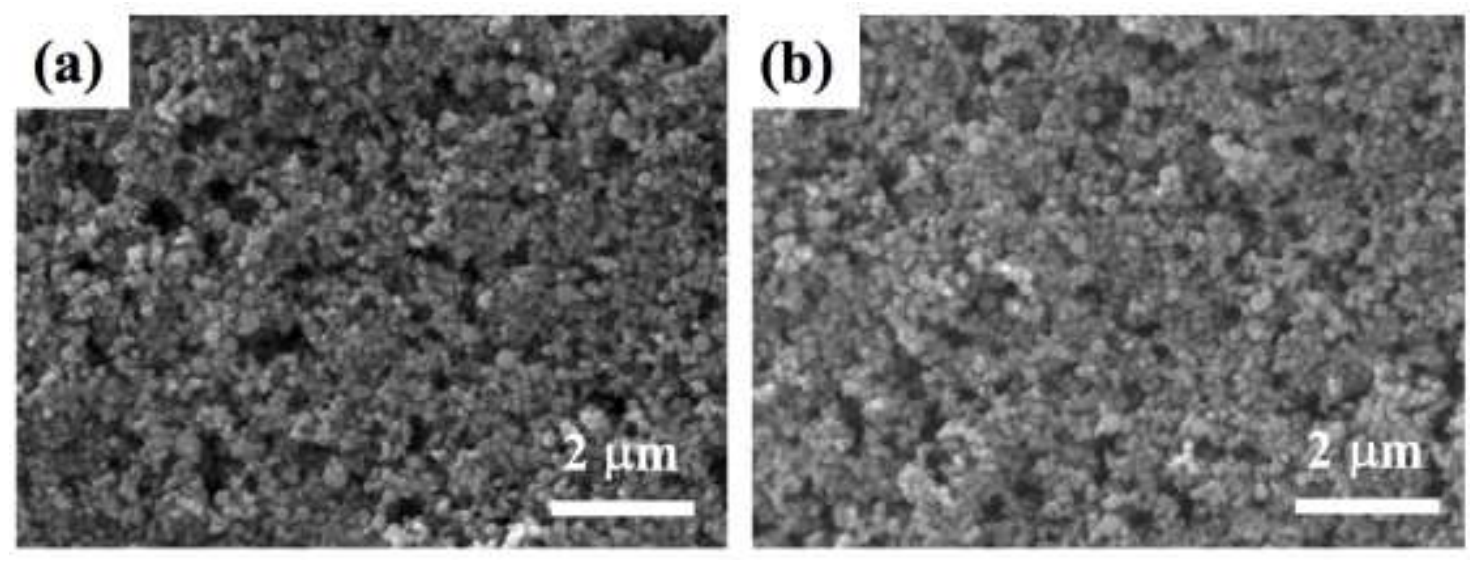

Figure S3. SEM images of the F-CuAg-00 films without formic acid. (a) before flash (b) after flash. 

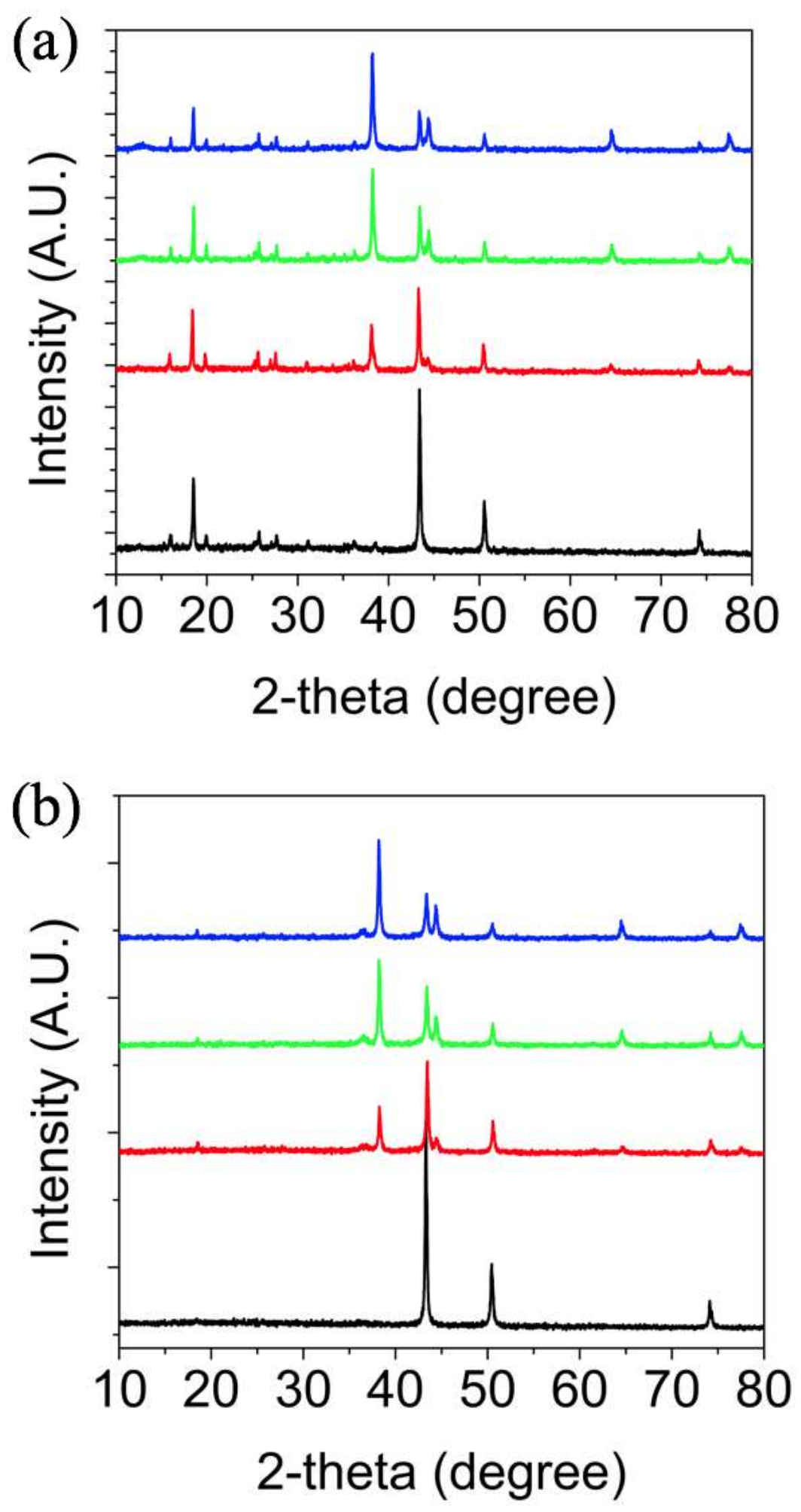
(c) XRD peaks and angles copper formate.

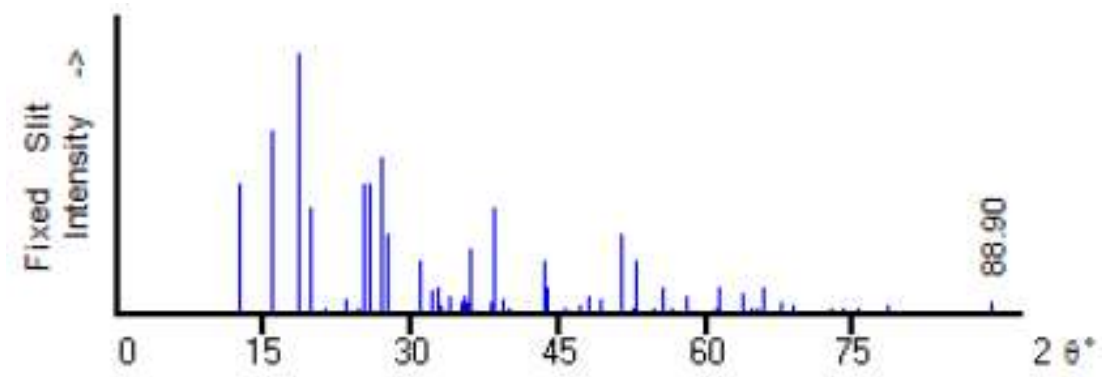

\begin{tabular}{|c|c|c|c|c|c|c|c|c|c|c|c|c|}
\hline 28 & Int-f & $\mathrm{h}$ & $\mathrm{k}$ & 28 & Int-f & $\mathrm{h}$ & $k$ & | & $2 \theta$ & Int-f & h & $k$ \\
\hline 12.474 & 50 & 2 & 00 & 38.453 & 40 & 3 & 3 & 1 & 61.108 & 2 & 2 & 5 \\
\hline 26 & 70 & 2 & 10 & 39.346 & 5 & 6 & 1 & 0 & 61.458 & 10 & & \\
\hline 18.429 & 100 & 1 & 11 & 40.057 & 2 & 3 & 2 & 2 & 62.279 & 1 & 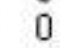 & 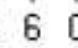 \\
\hline 19.845 & 40 & 0 & 20 & 42.109 & 1 & 4 & 3 & 1 & 63.692 & 7 & 7 & 4 \\
\hline 21.393 & 2 & 2 & 11 & 43.448 & 20 & 1 & 4 & 1 & 64.568 & 2 & 1 & 6 \\
\hline 452 & 5 & 2 & 20 & 43.624 & 8 & 4 & 2 & 2 & 65.362 & 2 & 6 & 5 \\
\hline 33 & 2 & 0 & 21 & 43.914 & 10 & 2 & 3 & 2 & 65.740 & 10 & 10 & 0 \\
\hline 20.0 & 50 & 4 & 00 & 45. & 2 & 6 & 2 & 1 & 67.528 & 4 & 3 & 6 \\
\hline 25.287 & 7 & 1 & 21 & 47.251 & 3 & 3 & 4 & 1 & 68.210 & 1 & 1 & 3 \\
\hline 25.620 & 50 & 3 & 11 & 48.209 & 6 & 7 & 1 & 1 & 68,460 & 1 & 8 & 3 \\
\hline 04 & 60 & 4 & 10 & 48.732 & 1 & 1 & 2 & 3 & 68.947 & 3 & 5 & 5 \\
\hline & 30 & 2 & 21 & 49.124 & 5 & 6 & 3 & 0 & & 2 & 6 & 5 \\
\hline 30.981 & 20 & 3 & 21 & 51.467 & 30 & 8 & 0 & 0 & 73.238 & 1 & 8 & 2 \\
\hline 59 & 8 & 4 & 20 & 52.258 & 1 & 3 & 2 & 3 & 73.891 & 2 & 10 & 3 \\
\hline 6 & 10 & 2 & 30 & 52.517 & 2 & 8 & 1 & 0 & 75.418 & 2 & 8 & 5 \\
\hline & 3 & 2 & 12 & 52.745 & 20 & 2 & 5 & 0 & 40 & 1 & 5 & 5 \\
\hline 87 & 6 & 1 & 31 & 54.229 & 1 & 5 & 4 & 1 & 78.576 & 3 & 11 & 2 \\
\hline 22 & 4 & 0 & 22 & 54.709 & 2 & 8 & 1 & 1 & 81,274 & 1 & 12 & 0 \\
\hline & 6 & 4 & 21 & 55.682 & 10 & 8 & 2 & 0 & 07 & 1 & 2 & 7 \\
\hline & 4 & 1 & 22 & 56.600 & 2 & 6 & 4 & 0 & 88.252 & 1 & 6 & 7 \\
\hline & 25 & 5 & 11 & 57. & 6 & 6 & 3 & 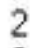 & 88.901 & 4 & 11 & 4 \\
\hline & 4 & 6 & 00 & 59.889 & 1 & 8 & 0 & & & & & \\
\hline
\end{tabular}

(d) XRD peaks and angles Ag.

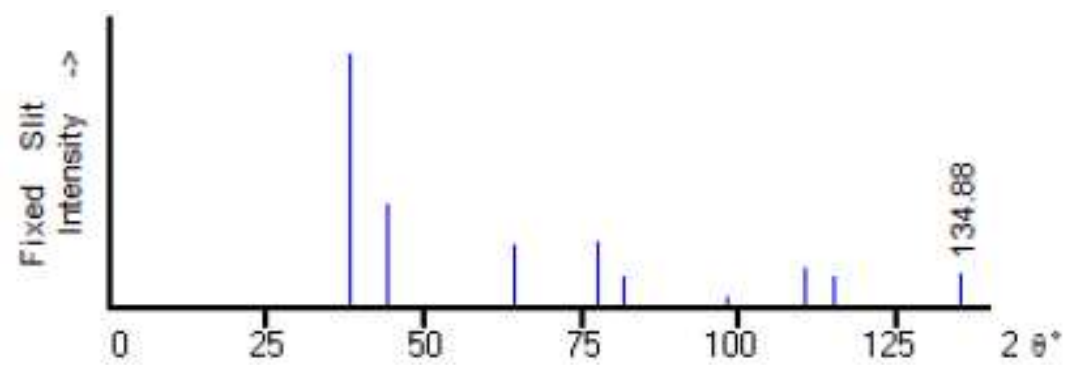

\begin{tabular}{lrlll|lrlll|lllll}
26 & Int-f & $h$ & $k$ & $l$ & 28 & Int-f & $h$ & $k$ & $l$ & 28 & Int-f & $h$ & $k$ & I \\
38.116 & 100 & 1 & 1 & 1 & 77.472 & 26 & 3 & 1 & 1 & 110.49 & 15 & 3 & 3 & 1 \\
44.277 & 40 & 2 & 0 & 0 & 81.537 & 12 & 2 & 2 & 2 & 114.92 & 12 & 4 & 2 & 0 \\
64.426 & 25 & 2 & 2 & 0 & 97.888 & 4 & 4 & 0 & 0 & 134.88 & 13 & 4 & 2 & 2
\end{tabular}


(e) XRD peaks and angles $\mathrm{Cu}$.

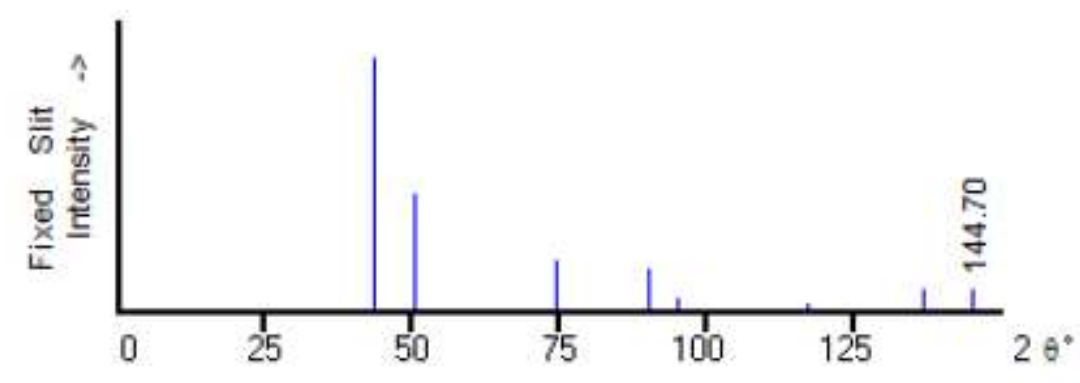

\begin{tabular}{lrlll|lrlll|lrrrr}
26 & Int-f & $h$ & $k$ & $l$ & 26 & Int-f & $h$ & $k$ & $l$ & 28 & Int $\cdot f$ & $h$ & $k$ & I \\
43.295 & 100 & 1 & 1 & 1 & 89.926 & 17 & 3 & 1 & 1 & 136.49 & 9 & 3 & 3 & 1 \\
50.431 & 46 & 2 & 0 & 0 & 95.135 & 5 & 2 & 2 & 2 & 144.70 & 8 & 4 & 2 & 0 \\
74.127 & 20 & 2 & 2 & 0 & 116.91 & 3 & 4 & 0 & 0 & & & & &
\end{tabular}

Figure S4. Full size graph of Figure 3: X-ray diffraction patterns (XRD) of the F-CuAg films with various concentrations of $\mathrm{AgNO}_{3}$ (Black: F-CuAg-00, red: F-CuAg-01, green: F-CuAg-02, and blue: F-CuAg-03); (a) before flash and (b) after flash (f: copper formate, $\mathrm{Cu}$ : metallic copper, and $\mathrm{Ag}$ : metallic silver). XRD reference peaks and angles of (c) copper formate, (d) silver, and (e) copper. 
(a)

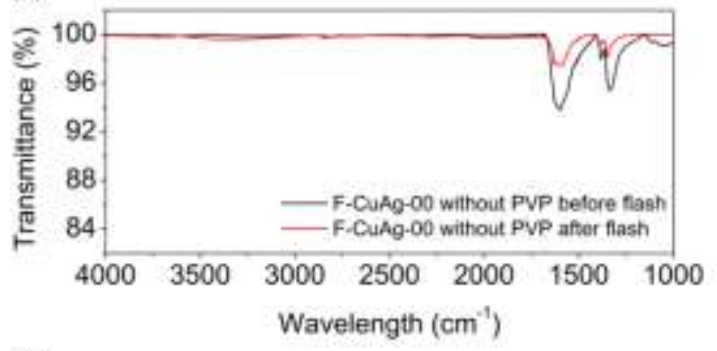

(c)

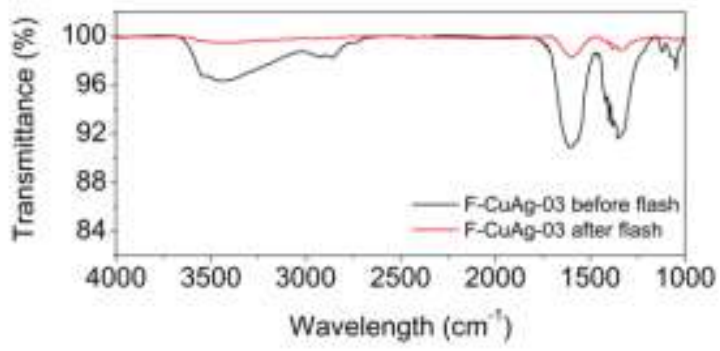

(b)

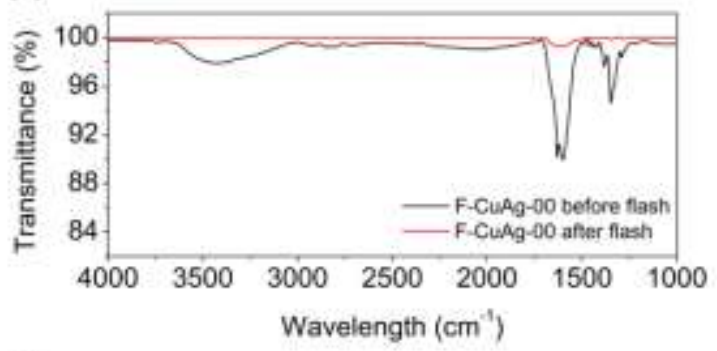

(d)

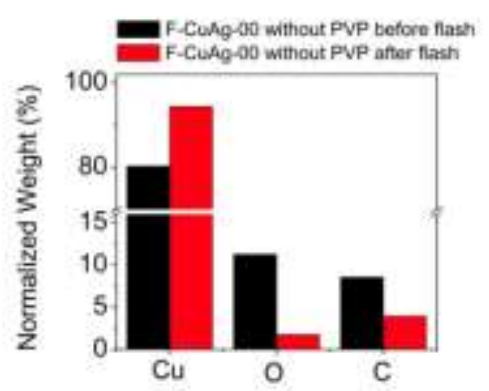

Figure S5. FT-IR spectrum of (a) F-CuAg-00 without PVP (b) F-CuAg-00 and (c) FCuAg-03. The PVP has FT-IR peaks at C-N: $1294 \mathrm{~cm}^{-1}, \mathrm{CH}_{3}: 1423 \mathrm{~cm}^{-1}, \mathrm{CH}_{2}: 1461$ $\mathrm{cm}^{-1}, \mathrm{C}=\mathrm{O}: 1652 \mathrm{~cm}^{-1}$ and $-\mathrm{OH}: 3410 \mathrm{~cm}^{-1}$. In addition, $1050 \mathrm{~cm}^{-1}, 1330 \mathrm{~cm}^{-1}$, and $1600 \mathrm{~cm}^{-1}$ are symmetric and asymmetric stretching in C-O of copper formate. Some peaks of PVP and copper formate are overlapped in this range. The carbon peaks were decreased after flash. (d) Normalized weight percent of $\mathrm{Cu}, \mathrm{O}$, and $\mathrm{C}$ of F-CuAg-00 without PVP which were obtained from EDS. Without PVP (Figure S5a), copper formate was decreased after flash, however, it was not fully converted which is shown in EDS (Figure S5d). 


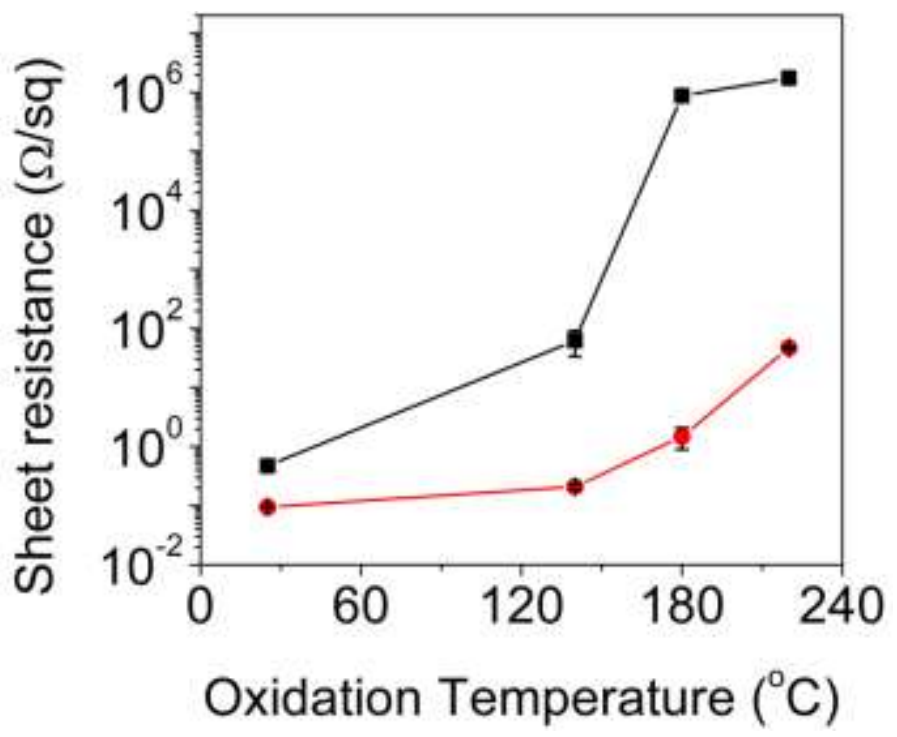

Figure S6. Sheet resistance as a function of the oxidation temperature at $90 \mathrm{~min}$ on FCuAg-00 (black) and F-CuAg-02 (red) films.

\section{Reference}

(1) Pulkkinen, P.; Shan, J.; Leppänen, K.; Känsäkoski, A.; Laiho, A.; Järn, M.; Tenhu, H. Poly(Ethylene Imine) and Tetraethylenepentamine as Protecting Agents for Metallic Copper Nanoparticles. ACS Appl. Mater. Interfaces 2009, 1 (2), 519-525. 